\title{
Power System Requirements
}

\author{
Edmund J. Conway
}

We estimated the electrical power required for each mission in the baseline model (fig. 1) and in the alternative model (fig. 2), according to the specific energy-using activities and operations shown. We then identified appropriate technologies to meet these power requirements, using such criteria as, Can the technology fully meet the requirement? and, Can the technology be ready at least 5 years before the mission? In some cases, there were competing technologies for the same mission.

\section{Low Earth Orbit (LEO)}

The initial space station, scheduled for the mid-1990s, will have 75-300 kW (electric) of continuous bus power. Mid- to late 1980s' solar photovoltaic technology is the only proven power-generating option available. However, solar photovoltaic systems require large arrays and consequently produce substantial drag. To provide power above the $75-\mathrm{kW}$ level, two technologies could compete: solar dynamic (solar thermal with
Stirling-, Brayton- or Rankine-cycle conversion) and nuclear thermal (with thermoelectric, thermionic, or dynamic conversion). Both technologies are now in developmental phases.

A second-generation space station appears in the baseline model at 2008. It would be needed for large-scale space processing of terrestrial materials. Space Station 2 would require from one to tens of megawatts. Such a mission would provide a major pull on the power-generating technologies. The current choice would appear to be some type of nuclear power system.

For power requirements above 1 megawatt, serious technology issues also arise in electrical power management (high voltage and current) and thermal management (how to dispose of $1 \mathrm{MW}$ of lowtemperature heat). Electrical power management would require both a new philosophy and some new technology. Thermal management would require such new technology as a large liquid droplet radiator. 


\section{Geosynchronous Earth Orbit (GEO)}

By the late 1990s, a geosynchronous experimental science platform would require up to $10 \mathrm{~kW}$. This requirement could be met by solar photovoltaic power. Advanced lightweight power generation and storage systems might be required if the present limitations on payload mass to GEO have not been eased significantly. Such systems, including those with gallium arsenide solar cells and high specific-power chemical storage, are in the research stage now.

By 2004 , a GEO shack or temporarily inhabited repair shop on the platform will allow for human-tended and interchangeable experiments. To operate in the repair shop, the human tenders would need additional power, on the order of $10 \mathrm{~kW}$. This power could be supplied by the visiting spacecraft. Solar photovoltaic technology, similar to that already mentioned for the platform, could be used.

A manned GEO station could be required beyond 2010 . The power level anticipated and the enabling technology are similar to those of the LEO growth space station.

Thus, geosynchronous Earth orbit provides no new power challenges. 


\section{Figure 3}

\section{Lunar Aover Used on the Apollo 17 Mission}

An automated unmanned version of this rover might be useful on future lunar missions. While seemingly simple, this Apollo Rover contained many of the elements necessary for a completely unmanned rover-a sophisticated redundant power system, power steering. automatic thermal control, a dust control system, and a self-contained navigation system which kept track of the location of the Rover at all times.

The Apollo 17 Rover, using two 36-volt silver-zinc batteries rated at 121 amphours each, traveled a maximum distance from the Lunar Module (LM) of $7.6 \mathrm{~km}$. For long unmanned traverses, battery power would probably not be practical because of the relatively low energy density of batteries.

A completely automated rover with an artificial intelligence (Al) system or a teleoperated rover are two possible versions for future applications.

\section{Moon}

An orbital lunar mapper in the midto late 1990s has only small power requirements, which can be met by 1980s' technology. An unmanned surface explorer (compare fig. 3), beginning in 2004 , would require only a few (2-5) kilowatts continuously, for movement, surface coring, analysis, and telemetry. A radioisotope generator (compare fig. 4) with dynamic conversion is the technology of choice.

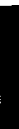

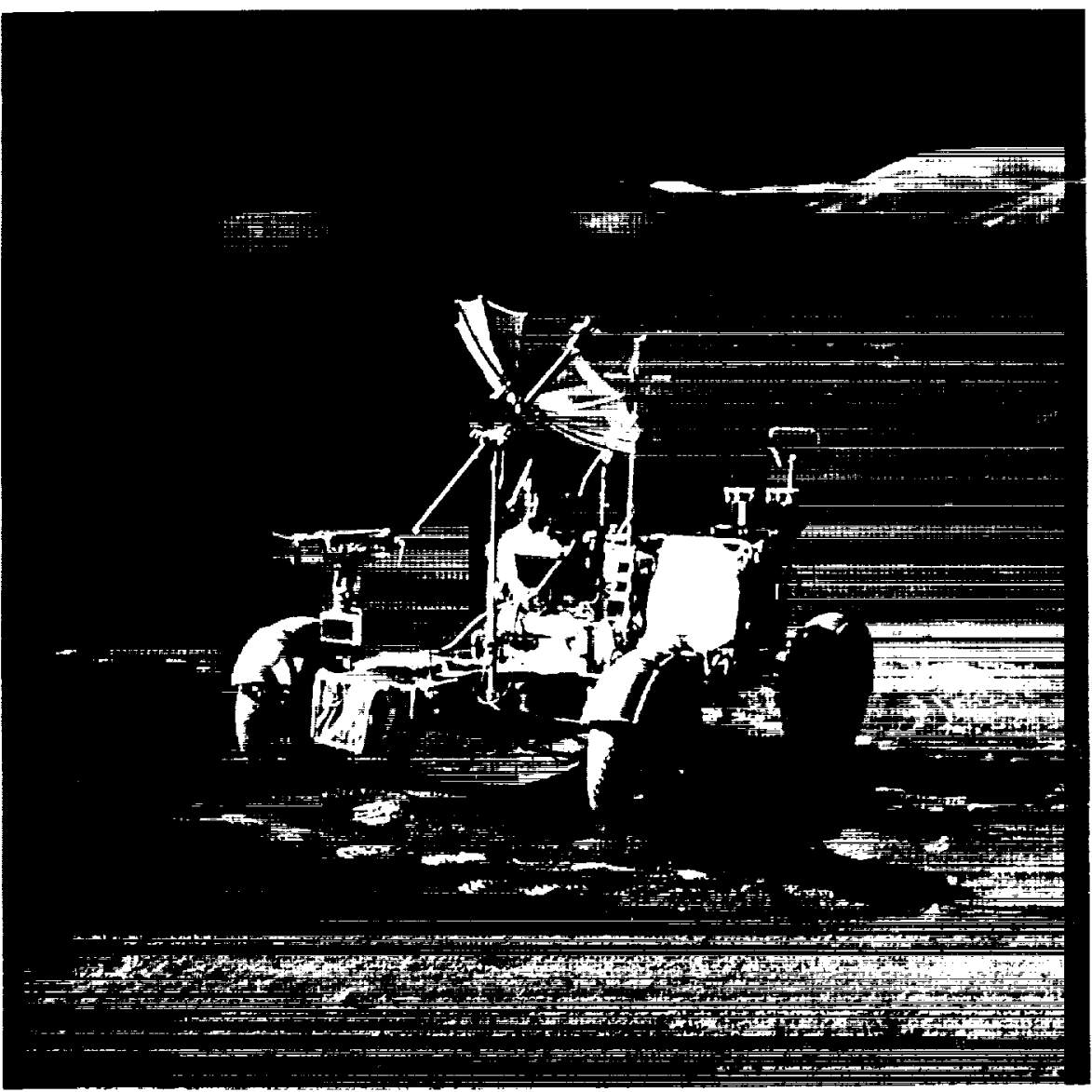



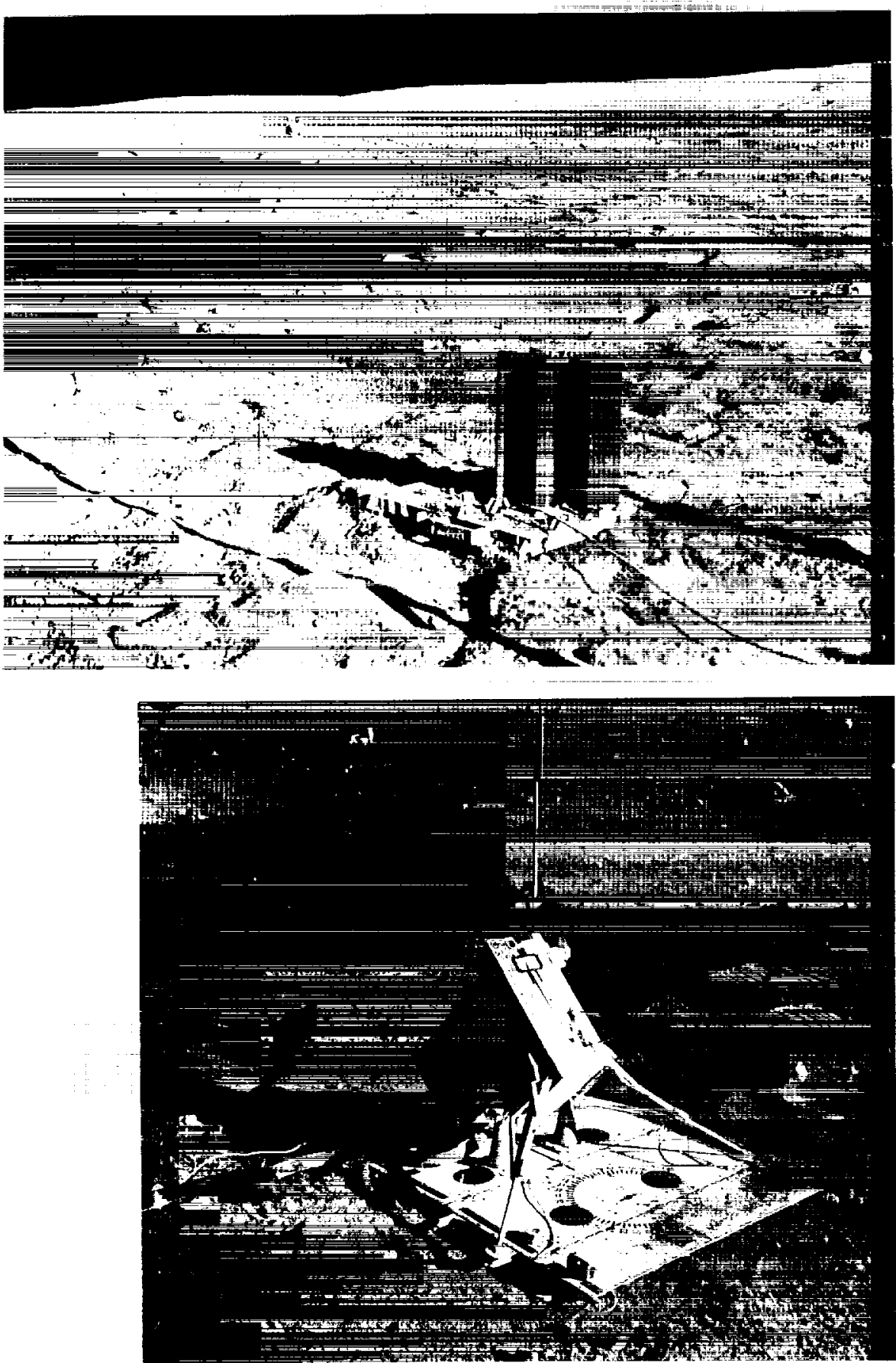

Figure 4

\section{a. Radioisotope Thermoelectric Generator}

This radioisotope thermoelectric generator (RTG) was the power source for the Apollo lunar surface experiments package (ALSEP) on the Apollo 16 mission. This power generator contains fins for radiating away excess heat. On this mission it powered an active seismic experiment (see accompanying fig.), a passive seismic experiment, a surface magnetometer, a heat flow experiment, and the central control and communications station.

b. Mortar Firing Assembly for the Active Seismic Experiment

This assembly in the ALSEP was designed to fire four grenades out to a maximum distance of $1.5 \mathrm{~km}$. The grenades were designed to explode on impact, generating a seismic signal which would be picked up by a string of three geophones. On the actual mission, only three of the grenades were used and the maximum distance traveled was about $900 \mathrm{~m}$. This experiment determined the thickness and seismic velocity of the near-surface structure at the Apollo 16 site. 
By 2010 , a lunar camp, to be inhabited only during the 2 weeks of lunar day, would initially require $25 \mathrm{~kW}$, supplied by a solar photovoltaic system. This initial power level could be augmented during future visits using similar or improved photovoltaic technology. Or the lunar camp's power system could grow, in the same manner as that of the space station, to include solar dynamic or nuclear supplies. The initial power level is suitable for crew life support, lunar science, and light work, but it does not provide the storable energy for heat and life support during the lunar night. For full-time habitation, the camp and later the base would rely on nuclear power supplying a few hundred kilowatts. (See the analogy in figure 5.) High power requirements away from the base for transportation or mining could be supplied by a separate source or by transmission. Point-to-point beamed transmission along the surface or between surface and space is possible.
Figure 5

\section{a. Spartan Lunar Base}

The early lunar base may consist of several modules similar to habitation and laboratory modules for the space station, which can be transported to the lunar surface and covered with lunar regolith for radiation protection. In many ways this early base would be like the American Station at the South Pole, which is probably the closest thing we have to a base on another planet.

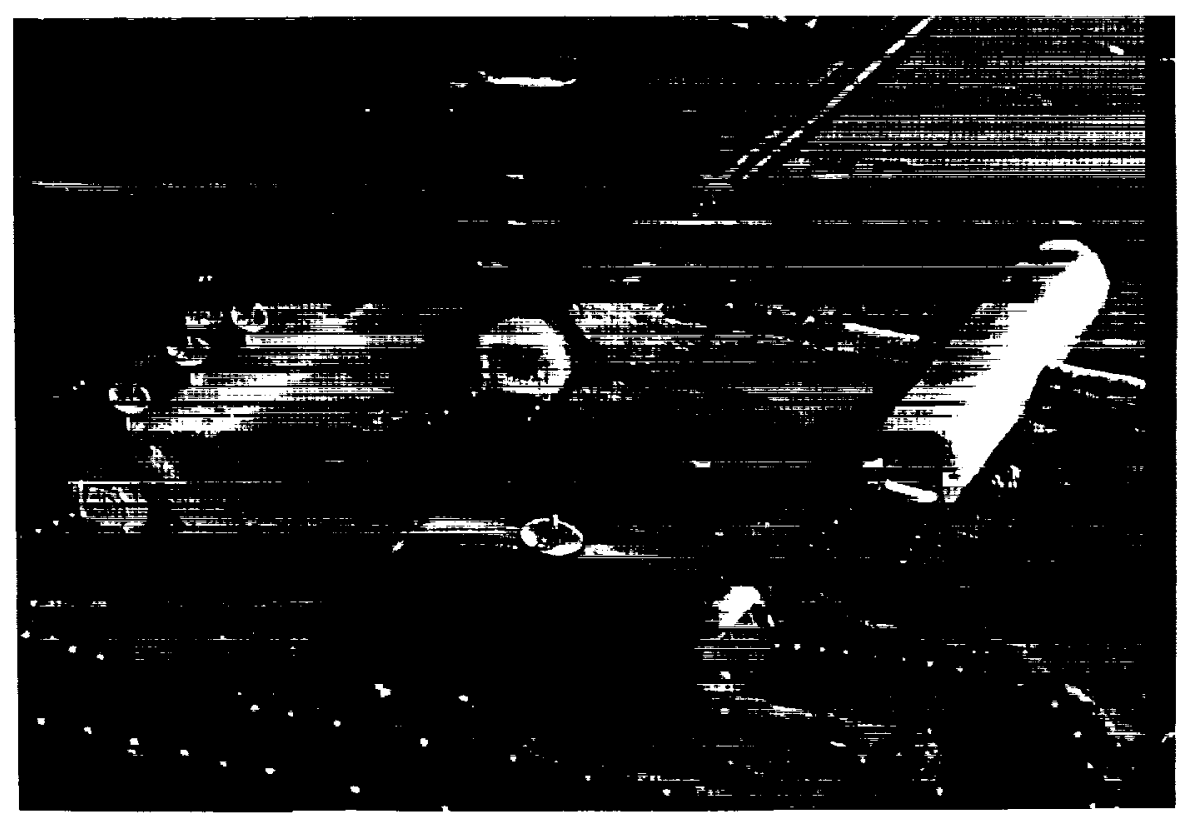




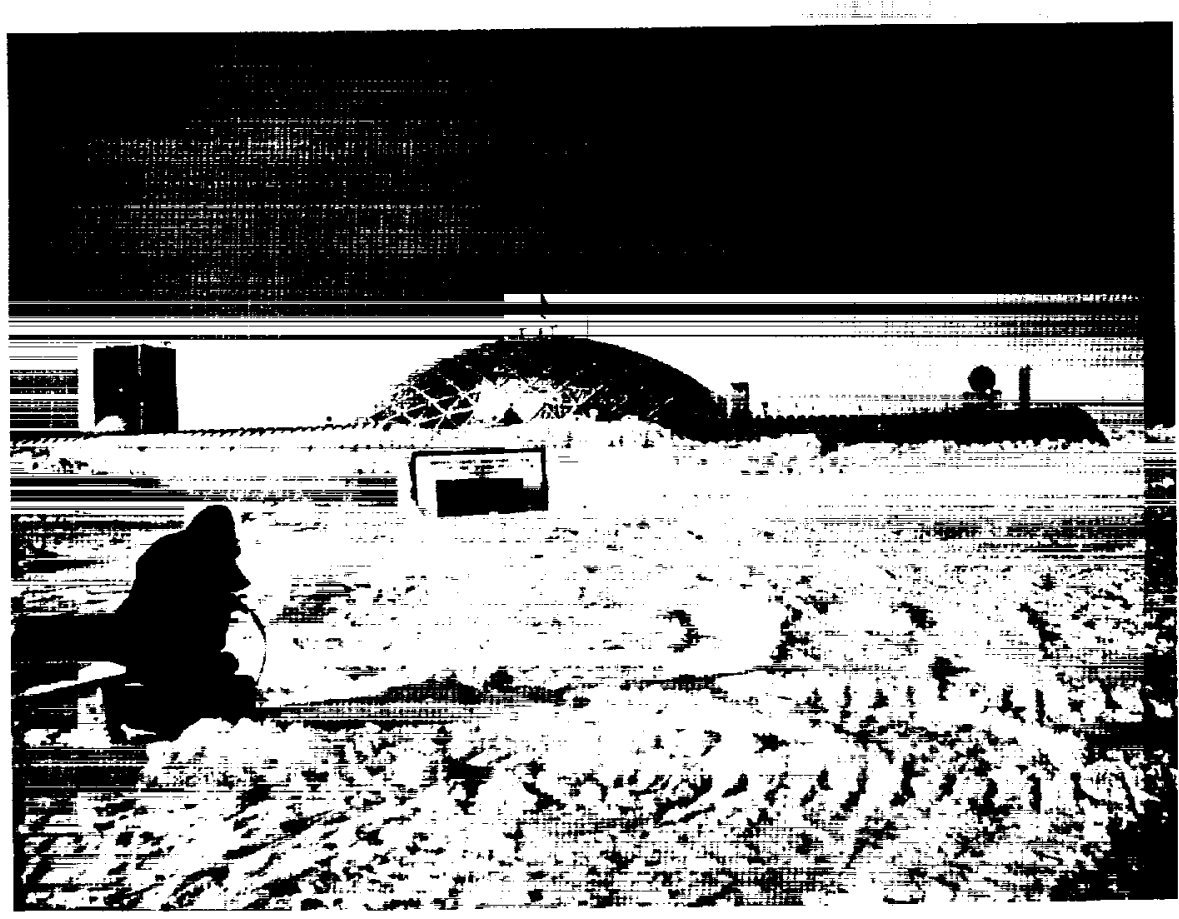

\section{b. South Pole Station}

The South Pole station is continuously occupied, but crewmembers arrive or depart only during the summer season. While the occupants can venture outside with protective clothing ("space suits") during the winter, they are mostly dependent on the shelter provided by the geodesic dome and the buildings within the dome, much as they would be at a Moon or Mars base.

Analogous to the Antarctic winter is the lunar night. More power would be required for heating and lighting in both cases. Even more important on the Moon, solar power would not be available at night unless massive storage was provided. Continuous occupation of a lunar base would probably rely on nuclear power.

Photo: Michael E. Zolensky

\author{
ORIGNAL FAGE \\ BLACK AND WHITE PHOTOGRAPH
}


Figure 6

\section{Unmanned Mars Lander}

In one concept (above), an unmanned Mars lander is bringing in a scientific package and ascent system while a small rover is parachuted to the surface in the distance. The rover could then travel to the lander in the foreground, collecting samples along the way. The rover would deliver the samples to the ascent system, which would take them into martian orbit and start them on their way back to Earth.

Much of the basic technology for such a mission was developed and successfully tested by the Viking lander (right). The Soviet Luna missions successfully refurned lunar samples to Earth in the early 1970s. Electrical power requirements for such missions are quite small compared to those for any manned mission.
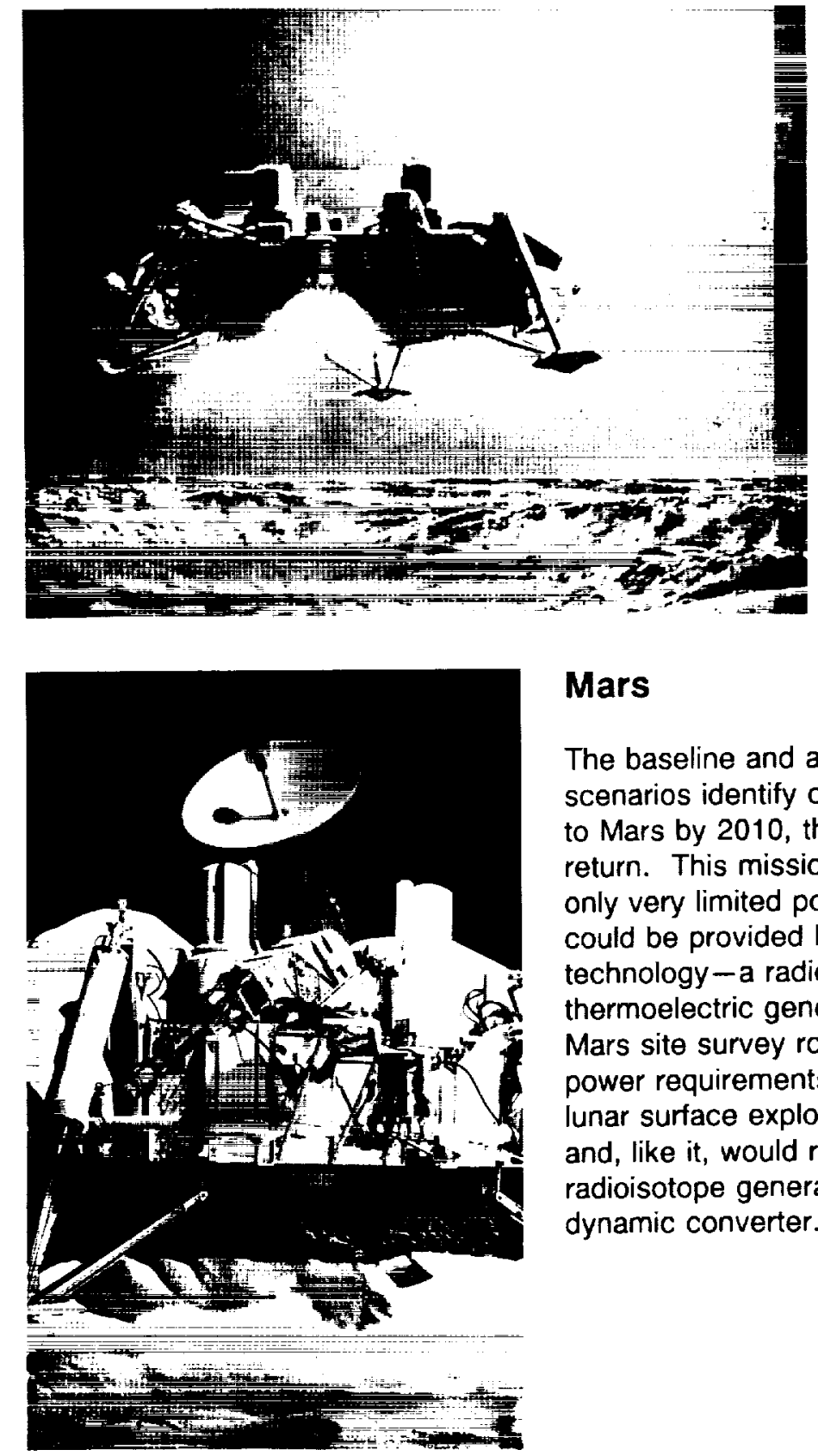

\section{Mars}

The baseline and alternative scenarios identify only one mission to Mars by 2010 , the Mars sample return. This mission would require only very limited power, which could be provided by current technology - a radioisotope thermoelectric generator. The later Mars site survey rover would have power requirements similar to the lunar surface explorer $(2-5 \mathrm{~kW})$ and, like it, would rely on a radioisotope generator with a dynamic converter. (See figure 6.) 


\section{Asteroids}

The alternative model (fig. 2) includes unmanned exploration of an asteroid beginning in 2005 . This involves activities and power requirements similar to those for the earlier lunar surface explorer and could be handled by a similar system.
Mining (not included in the scenario) would require power on the order of $10 \mathrm{MW}$. A nuclear reactor power system developed for general application to industrial processing in space would be utilized. See figure 7 for a medium-range application on one of the asteroid-like moons of Mars.

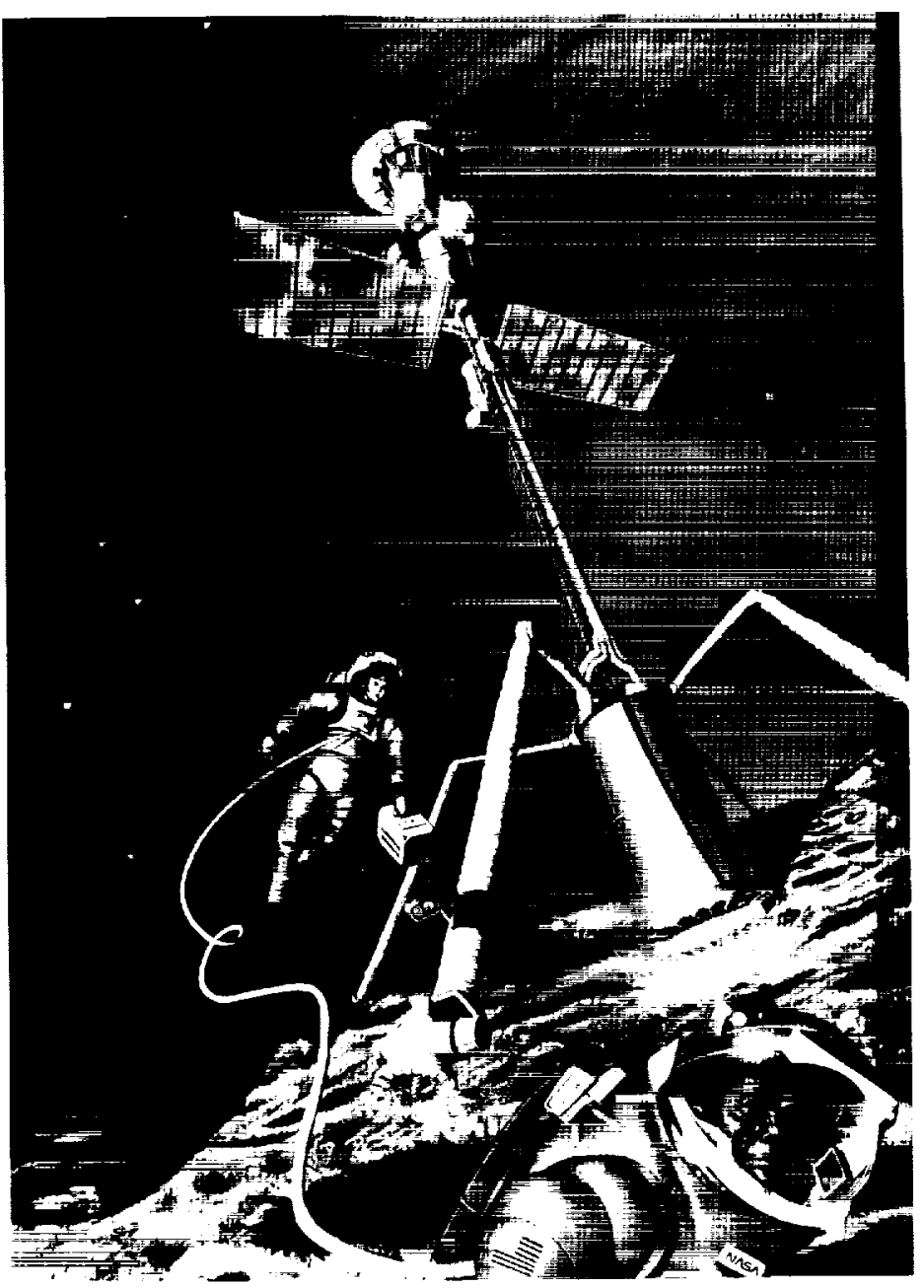

Figure 7

\section{Phobos Deimos Hot Drill}

The Phobos/Deimos (PhD) "hot drill" is designed to melt its way into the regolith of one or the other of these satellites. liberating volatiles (mainly water) as it goes. Water could be trapped and electrolyzed into hydrogen and oxygen for use as propellants to refuel the martian lander or the Earth-Mars vehicle.

Artist Pat Rawlings 